\title{
A Fingerprint-based Age and Gender Detector System using Fingerprint Pattern Analysis
}

\author{
A. S. Falohun \\ Dept. of Computer Sc. \& Eng. \\ Ladoke Akintola Univ. of Tech. \\ Ogbomoso, Nigeria.
}

\author{
O. D. Fenwa \\ Dept. of Computer Sc. \& Eng. \\ Ladoke Akintola Univ. of Tech. \\ Tech. \\ Ogbomoso, Nigeria.
}

\author{
F. A. Ajala \\ Dept. of Computer Sc. \& Eng. \\ Ladoke Akintola Univ. of \\ Ogbomoso, Nigeria.
}

\begin{abstract}
Humans have distinctive and unique traits which can be used to distinguish them thus, acting as a form of identification. Biometrics identify people by measuring some aspect of individual's anatomy or physiology such as hand geometry or fingerprint which consists of a pattern of interleaved ridges and valleys. The year 2015 election in Nigeria was greeted by some petitions including under-aged voters. The need for an age and gender detector system is a major concern for organizations at all levels where integrity of information cannot be compromised. This work developed a system that determines human age-range and gender using fingerprint analysis trained with Back Propagation Neural Network (for gender classification) and DWT+PCA (for age classification). A total of 280 fingerprint samples of people with various age and gender were collected. 140 of these samples were used for training the system's Database; 70 males and 70 females respectively. This was done for age groups 1-10, 11-20, 21$30,31-40,41-50,51-60$ and 61-70 accordingly. In order to determine the gender of an individual, the Ridge Thickness Valley Thickness Ratio (RTVTR) of the person was put into consideration. Result showed $80.00 \%$ classification accuracy for females and $72.86 \%$ for males while 115 subjects out of $140(82.14 \%)$ were correctly classified in age.
\end{abstract}

\section{General Terms}

Fingerprint, Pattern Recognition, Biometric, Classification.

\section{Keywords}

Authentication, Histogram equalization, Ridge, Gender, Age.

\section{INTRODUCTION}

Biometric technology that involves the identification and verification of individuals by analysing the human body characteristics such as the finger prints, palm vein, iris and the likes has been widely used in various aspect of life for different purposes including the voting system. In recent time, there has been high level of acclaimed under-aged voters registration and under-aged voters which in turn marred electioneering particularly in developing countries resulting in election irregularities. Also observed is the idea of impersonation experienced on a daily basis in both private and public sectors, the ghost worker syndrome which has become a menace across all tiers of government in underdeveloped and developing countries. Despite the numerous advantages of the biometric system and its impact to various work sectors across the globe, most biometric technology users face the issue of defining the right and accurate biometric technology system that will be cost effective in solving particular problems in specific environment. Fingerprints are a form of biometric identification which is unique and does not change in one's entire lifetime; this is being applied in an electronic voting system like what obtained in the Nigerian 2015 States and Presidential elections. In the context of information systems, a general definition of a biometric system is that of, "a pattern recognition system that recognizes a person by determining the authenticity of a specific physiological and/or behavioral characteristic possessed by that person" [15], [4]. The earliest documented use of systematic biometric methods as a means to distinguish one individual from another can be traced back to $14^{\text {th }}$ century China. Chinese merchants were known to stamp children's inked palm and footprints on paper in order to tell them apart. In western culture, the earliest documented distinction made of a biometric property was that done by the English botanist Nehemiah Grew, who in 1684, published a paper reporting the distinct characteristics of ridge, furrow and pore structures in human fingerprints.

Fingerprints are fully formed (i.e. became stable) at nine months of foetus development and finger ridge configurations do not change throughout the life of an individual, except in case of accidents such as cuts on the fingertips which lead to reduction in quality index of finger image in the image taken and image processing in the anticipated subsequent stages of fingerprint analysis. Injuries like cuts, burns and bruises can temporarily damage quality of fingerprints but when fully healed, patterns will be restored. This property of fingerprint makes it a very attractive biometric identifier [2]. Although soft biometric characteristics, gender and age for example, are not discriminative enough to distinguish individuals, they provide additional evidence that may complement primary biometric identifiers such as fingerprints, faces, irises [9]. Therefore, they can contribute to recognition accuracy.

\section{LITERATURE REVIEW}

\subsection{Origin of Biometrics}

[11] gave an extensive overview on the origin of biometrics traceable to recognition techniques developed over thousands of years now by the people of ancient Egypt and China. The author cited the first recorded systematic capture of hand and finger images (1858) for identification purposes used by Sir William Herschel, Civil Service of India, as a recorded handprint on the back of a contract for each worker to distinguish employees [13]. Also, State University of New York at Canton (2003) reported that during 1870, Alphonse Bertillon developed a method of identifying individuals based on detailed records of their body measurements, physical descriptions and photographs. This method was termed 
"Bertillonage" or anthropometrics and the usage was aborted in 1903 when it was discovered that some people shared same measurements and physical characteristics.

Human fingerprints have been discovered on a large number of archeological artifacts and histological items. Although these findings provide evidence to show that ancient people were aware of the individuality of fingerprints, it was not until the late sixteenth century that the modern scientific fingerprint technique was first initiated [12]. In 1686, Marcello Malpighi, a professor of anatomy at the University of Bologna noted in his writings the presence of ridges, spirals and loops in fingerprints. Since then, a large number of researchers have invested huge amounts of effort on fingerprint studies [8].

\subsection{Types of fingerprint pattern}

Before computerization replaced manual filing systems in large fingerprint operations, manual fingerprint classification systems were used to categorise fingerprints based on general ridge formation (such as the absence or presence of circular patterns in various fingers).

The three basic patterns of fingerprint ridges are arch, loop and whorl.

Arch is a pattern where the ridges enter from one side of the finger, rise in the center forming an arc, and then exit the other side of the finger.

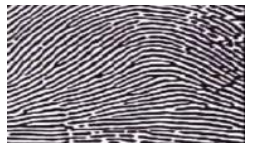

Plain arch pattern

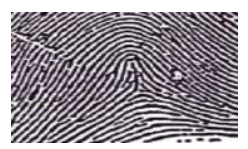

Tented Arch pattern
Figure 1a: types of arch pattern

Loop is a pattern where the ridges enter from one side of a finger, from a curve, and tend to exit from the same side enter

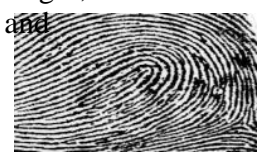

Radial loop pattern

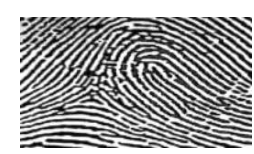

Ulnar loop pattern
Figure 1b: types of loop pattern

Whorl is a pattern where ridges form circulatory around a central point on the finger. Scientists have found that family members often share the same general fingerprint patterns, to the belief that these patterns are inherited.
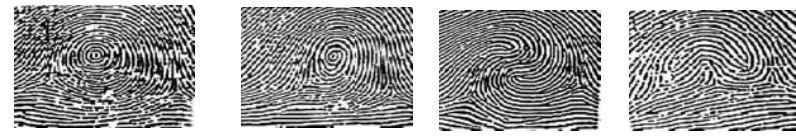

Plain whorl

Central pocket

Double loop

Accidental whorl

Figure 1c: types of whorl pattern. (Source: [6]).

[7] developed a system to verify and authenticate students in higher institutions of learning for class attendance using fingerprint pattern analysis.

Fingerprint identification and classification has been extensively researched in times past, however very few researchers have studied the fingerprint gender classification problem. [1] used the ridge density, which he defined as the number of ridges in a certain space; it was shown that the females have higher ridge density. [1]. [14] also established that the males have higher ridge breadth,) which was defined as the distance between the centers of two adjacent valleys), than females.

Two studies revealed that the males have higher ridge count than the females [9], [5]. It was also shown that both males and females have higher rightward directional asymmetry in the ridge count, with the asymmetry being higher in males than females [9] and higher incidence of leftward asymmetry in females. [5]. Female's fingerprints are significantly of lower quality than male fingerprints [10].

[16] reported the findings of [3] establishing that the female's fingerprint is characterized by a high Ridge Thickness Valley Thickness Ratio, while the male's fingerprint is characterized by low Ridge Thickness Valley Thickness Ratio, with the exception of small percentage of male's fingerprints having high Ridge Thickness Valley Thickness Ratio, and female's fingerprints having low Ridge Thickness Valley Thickness Ratio.

[16] presented a model towards the determination of gender through the fingerprint information using Multi-Layer Perceptron (MLP) technique and results for age and gender showed also that females have a higher ridge thickness to valley thickness ratio compared to males with $80 \%$ gender accuracy and $33.33 \%$ age accuracy.

\section{METHODOLOGY}

\subsection{Fingerprint acquisition/Data collection}

Each of the 10 fingerprints of 280 subjects were captured using the fingerprint biometric optical sensor, SECUGEN SDU03P with its SDK interfaced to the system through the USB. The age was grouped in 10 years in 7 categories (i.e 1$10,11-20,21-30 . ., 61-70)$.

\subsection{Enhancement}

The performance of minutiae extraction algorithms and fingerprint recognition technique relies heavily on the quality of the input fingerprint images. Since the fingerprint images acquired from sensors or other media are not assured with perfect quality; In practice, due to skin conditions (e.g. wet or dry, cuts and bruises), sensor noise, incorrect finger pressure, and inherently low quality fingers (e.g. elderly people, manual workers), a significant percentage of fingerprint images is of poor quality. The importance of fingerprint enhancement algorithm is to improve the clarity of the ridge structures in the recoverable region and mark the unrecoverable region for further processing. Here, Histogram equalization was employed.

\subsection{Image Binarization}

Fingerprint Image Binarization is to transform the 8-bit Gray fingerprint image (Gray-scale) to a 1-bit image with 0 -value for ridges and 1-value for valleys (binary). After the operation, ridges in the fingerprint are highlighted with black color while valleys are white. A locally adaptive binarization method is performed to binarize the fingerprint image. Such a named method comes from the mechanism of transforming a pixel value to 1 if the value is larger than the mean intensity value of the current block to which the pixel belongs.

\subsection{Segmentation}

Only a Region of Interest (ROI) is useful to be recognized for each fingerprint image. The image area without effective ridges and valleys is first discarded since it only holds background information. Then the bound of the remaining effective area is sketched out since the minutiae in the bound region are confusing with those spurious minutiae that are generated when the ridges are out of the sensor. Locating the reference point i.e., ROI is an essential step that can influence 
the matching accuracy. Two major methods are commonly employed, one is Complex filters and the other is Poincare index analysis. The anomaly associated with Poincare index is that it does not detect the arch type fingerprint. So in the course of this work, Complex filters was used.

\subsection{Fingerprint Ridge Thinning}

Ridge Thinning is to eliminate the redundant pixels of ridges till the ridges are just one pixel wide. An iterative, parallel thinning algorithm was used. In each scan of the full fingerprint image, the algorithm marks down redundant pixels in each small image window (3x3). And finally removes all those marked pixels after several scans.

\subsection{Minutia Marking}

After the fingerprint ridge thinning, marking minutia points were identified, Crossing Number $(\mathrm{CN})$ concept was used for minutiae extraction

3.7 Training was done with Back Propagation Neural Network.

\subsection{DWT/PCA Testing}

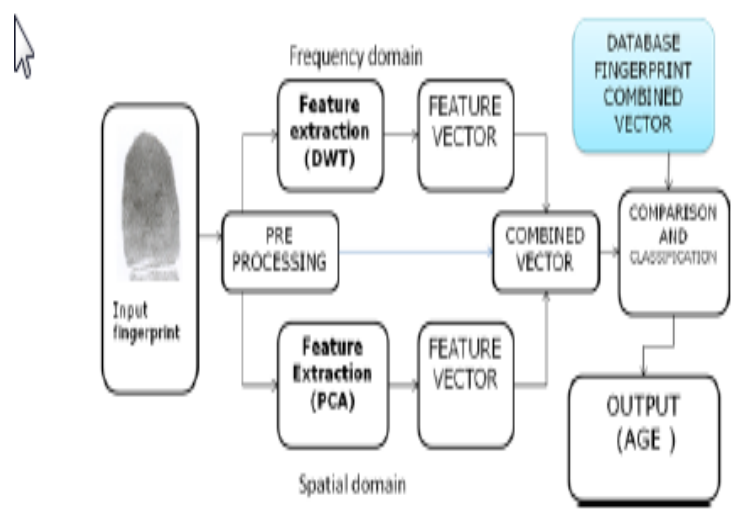

Figure 2: Flow chart showing DWT/PCA Testing

\subsection{Creating the Application}

The Application was created using MatLab. Integrated Development Environment.

\section{Artificial Neural Network (ANN)}

Prior to classification at all, the network needs to be trained. The neural network training done is as shown in Figure 3 while Figures $4 a-4 d$ showed the classification interfaces.

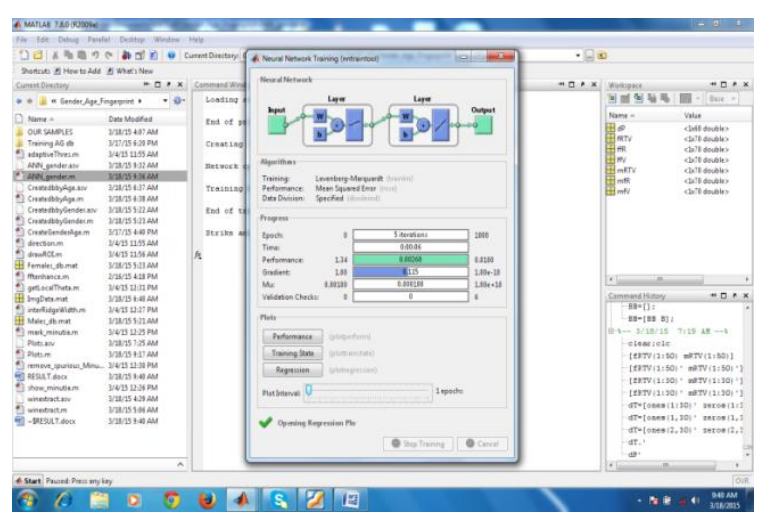

Figure 3 Network Page

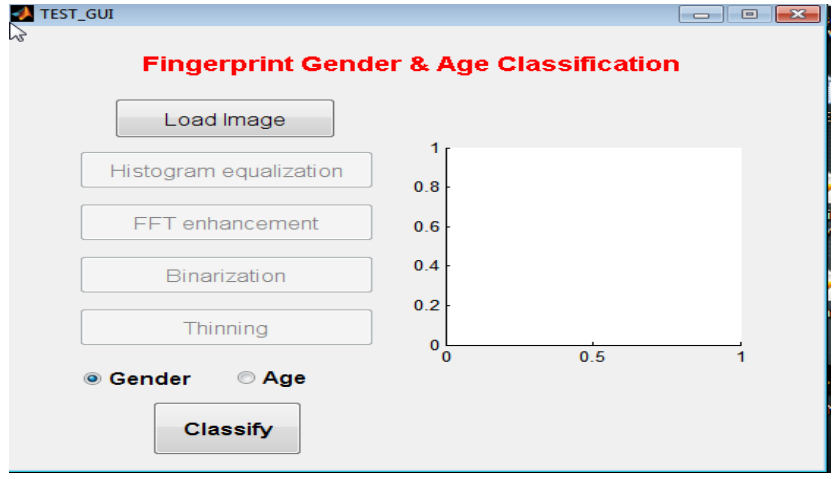

Figure 4a. Classification Interface

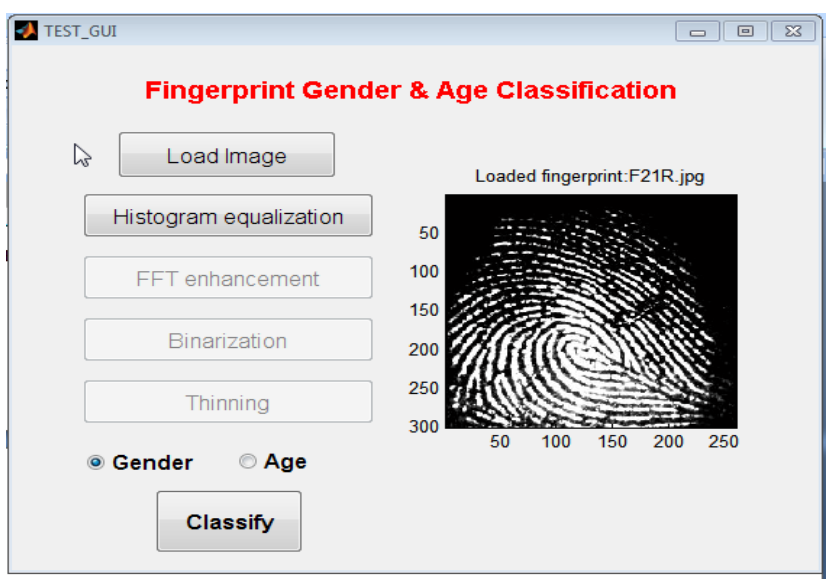

Figure 4b: Probe Fingerprint on Testing

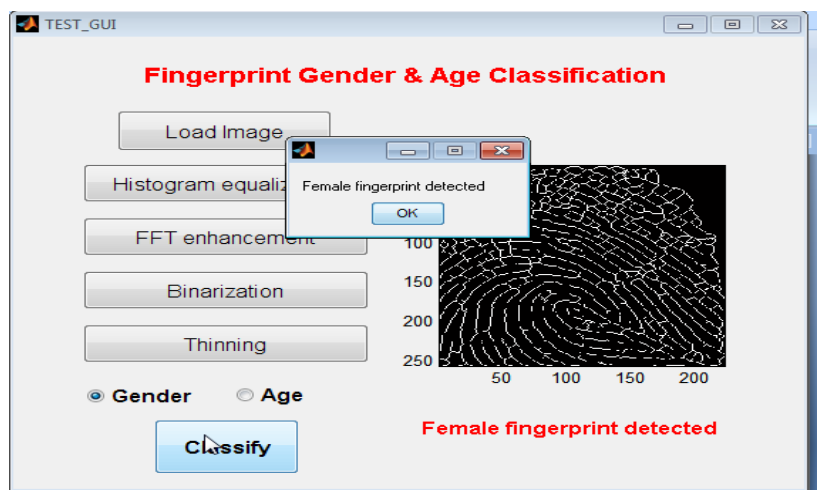

Figure 4c: Classification Interfaces showing a Female Gender

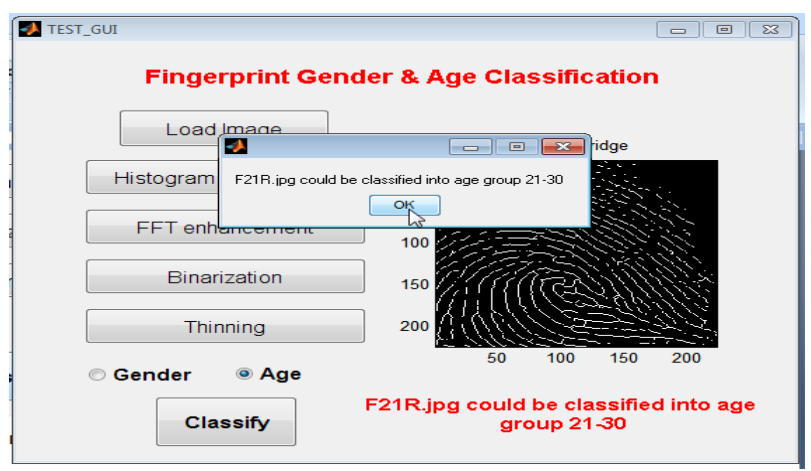

Figure 4d: Classification Interface Showing the Age Range of a Person 


\section{IMPLEMENTATION RESULTS ANDDISCUSSION}

\subsection{Analysis of results}

The network having been trained and tested with data (the acquired fingerprint samples) as shown in figures 3 and 4(ad), the following results were obtained and presented.

\subsection{Gender Classification}

Table 1: Female and Male Ridge counts, Valley counts and their Ratio

\begin{tabular}{|l|l|l|l|l|l|l|}
\hline & \multicolumn{2}{|l|}{ Ridge count } & \multicolumn{2}{l|}{ Valley count } & \multicolumn{2}{l|}{ Ridge/ Valley } \\
\hline $\begin{array}{l}\text { FP } \\
\text { No }\end{array}$ & Male & $\begin{array}{l}\text { Fem } \\
\text { ale }\end{array}$ & Male & $\begin{array}{l}\text { Femal } \\
\text { e }\end{array}$ & Male & $\begin{array}{l}\text { Femal } \\
\text { e }\end{array}$ \\
\hline 1 & 4390 & 3885 & 45786 & 32723 & 0.0959 & 0.1187 \\
\hline 2 & 4390 & 4866 & 45786 & 45310 & 0.0959 & 0.1074 \\
\hline 3 & 6044 & 6096 & 44132 & 40496 & 0.1370 & 0.1505 \\
\hline 4 & 4217 & 5904 & 42375 & 40688 & 0.0995 & 0.1451 \\
\hline 5 & 4217 & 5904 & 42375 & 40688 & 0.0995 & 0.1451 \\
\hline
\end{tabular}

\begin{tabular}{|l|l|l|l|l|l|l|}
\hline 6 & 4226 & 5573 & 35710 & 44603 & 0.1183 & 0.1249 \\
\hline 7 & 4270 & 5573 & 45906 & 44603 & 0.0930 & 0.1249 \\
\hline 8 & 4270 & 4639 & 45906 & 41953 & 0.0930 & 0.1106 \\
\hline 9 & 4270 & 4639 & 45906 & 41953 & 0.0930 & 0.1106 \\
\hline 10 & 4270 & 4639 & 45906 & 41953 & 0.0930 & 0.1106 \\
\hline 11 & 5061 & 4848 & 45115 & 45328 & 0.1122 & 0.1070 \\
\hline 12 & 4494 & 4545 & 45682 & 45631 & 0.0984 & 0.0996 \\
\hline 13 & 3946 & 3832 & 46230 & 46344 & 0.0854 & 0.0827 \\
\hline 14 & 4578 & 5122 & 45598 & 45054 & 0.1004 & 0.1137 \\
\hline 15 & 4495 & 4139 & 45681 & 42453 & 0.0984 & 0.0975 \\
\hline
\end{tabular}

Table 2 RTVTR RESULT

\begin{tabular}{|l|l|l|l|l|l|l|l|l|l|l|l|l|l|l|l|}
\hline S/N & 1 & 2 & 3 & 4 & 5 & 6 & 7 & 8 & 9 & 10 & 11 & 12 & 13 & 14 & 15 \\
\hline M & 0.105 & 0.12 & 0.07 & 0.1 & 0.09 & 0.08 & 0.09 & 0.1 & 0.11 & 0.11 & 0.1 & 0.12 & 0.1 & 0.12 & 0.07 \\
\hline F & 0.12 & 0.1 & 0.16 & 0.13 & 0.13 & 0.13 & 0.13 & 0.13 & 0.125 & 0.1 & 0.11 & 0.1 & 0.14 & 0.09 & 0.15 \\
\hline
\end{tabular}

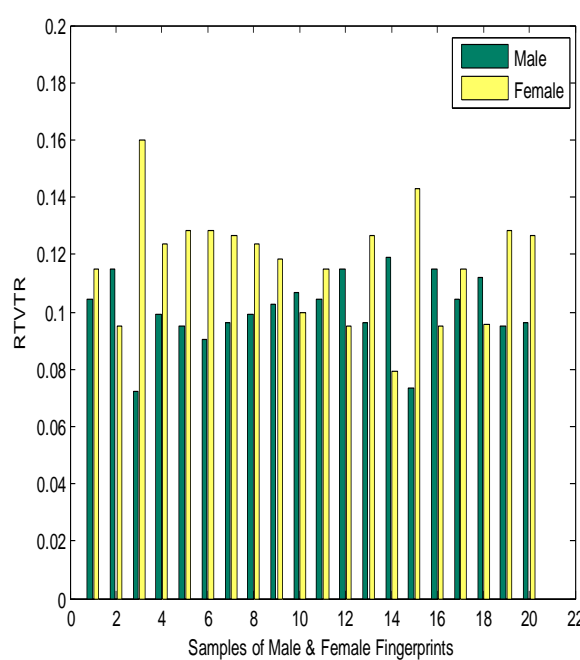

Figure 5: RTVTR for Male and Female

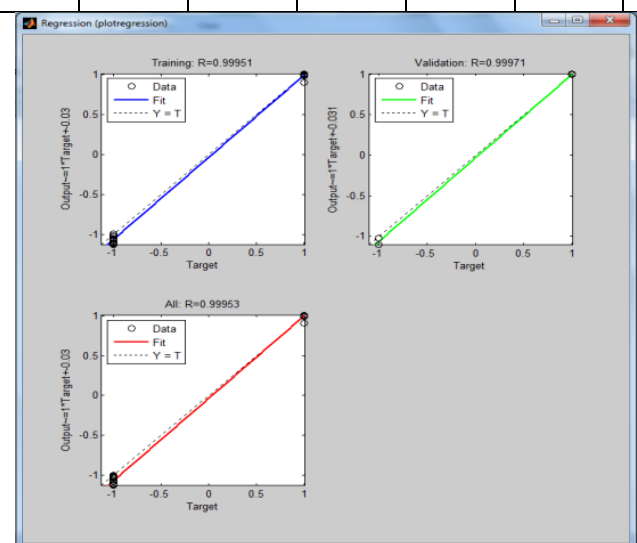

Figure 6: Parameters of the ANN model

\section{Performance}

The performance goal of the ANN was set to train the ANN model until the training error (MSE) falls below 10-2. As it is shown in the graph, the "Train" and "Validation" lines cross the 10-2 downwards.

The performance is best read by viewing the exact point where 'Validation' reaches the 'Best'. Validation was at best goal where MSE cuts the epoch i.e., at 0.95511 for MSE and at 5epochs. Performance goal was set to 1e-2 (10-2) and as it was shown in the figure(mk787) where the training network reached a reasonable target of 0.99951 while the validation 
reaches 0.99971 indicating that the trained and tested (almost the same) came very close.

Table 3 is a table formed comparing the actual input and the NN outputs of 12 samples of people: 6 females and 6 males. "Actual" Female fingers are labeled as 1 while "Actual" male fingers are labeled as 0 . The 3rd and the 4th showed the best result for females (closest to 1), the 9th showed the best for male (closest to 0) while the 6th showed the worst result in the table misclassifying a female as a male.

However, generally speaking, the females were recognized with ANN feature codes close to 1 while those for males are closing in on 0.At the end of the BPNN process it was shown that the method proved satisfactory.

Table 3: NN Outputs

\begin{tabular}{|l|l|l|l|l|}
\hline S/N & Actual & $\begin{array}{l}\text { Actual } \\
\text { Gender }\end{array}$ & Predicted & $\begin{array}{l}\text { Predicted } \\
\text { Gender }\end{array}$ \\
\hline 1 & 1 & F & 1.00268 & F \\
\hline 2 & 1 & F & 1.00268 & F \\
\hline 3 & 1 & F & 1.00148 & F \\
\hline 4 & 1 & F & 1.00148 & F \\
\hline 5 & 1 & F & 0.95115 & F \\
\hline 6 & 1 & F & -0.03538 & M \\
\hline 7 & 0 & M & -0.03538 & M \\
\hline 8 & 0 & M & -0.00389 & M \\
\hline 9 & 0 & M & 0.08123 & M \\
\hline 10 & 0 & M & 0.10941 & M \\
\hline 11 & 0 & M & 0.18057 & M \\
\hline 12 & 0 & M & 0.24863 & M \\
\hline
\end{tabular}

\subsection{Age classification}

It should be recalled that ANN/BPNN was not used for the age classification because reference materials and the existing works have shown that the ANN/BPNN don't work well for age classification, so DWT+PCA was used. The minimum distance of the query vectors (testing images) and the trained are compared, and the class with the closest distance was classified. 35 samples of fingerprint images were taken from each age group; 5 from each range and the result are as shown in table 3.

Table 3: Age Classification Results

\begin{tabular}{|l|c|c|}
\hline $\begin{array}{l}\text { Age } \\
\text { Bracket }\end{array}$ & $\begin{array}{l}\text { Correct } \\
\text { Classification }\end{array}$ & $\begin{array}{l}\text { Incorrect } \\
\text { Classification }\end{array}$ \\
\hline $1-10$ & 5 & 0 \\
\hline $11-20$ & 3 & 2 \\
\hline $21-30$ & 5 & 0 \\
\hline
\end{tabular}

\begin{tabular}{|l|c|c|}
\hline $31-40$ & 3 & 2 \\
\hline $41-50$ & 4 & 1 \\
\hline $51-60$ & 4 & 1 \\
\hline $61-70$ & 3 & 2 \\
\hline
\end{tabular}

Table 3: Percentage Accuracy of the System

\begin{tabular}{|c|c|}
\hline GENDER & AGE \\
ACCURACY & ACCURACY \\
\hline FEMALES : $80.00 \%$ & \\
MALES : $72.86 \%$ & $82.14 \%$ \\
\hline
\end{tabular}

\section{DISCUSSION OF RESULTS}

From the experiments conducted and sampled results presented, the following holds.

(1) Females have higher RTVTR than males.

56 females out of 70 were correctly recognized while 51 of 70 males were duly recognized.

(2) Out of 140 tested, a total of 115 were correctly classified in age leaving out 25 subjects.

(3) Observation showed that there was an overlap between the different age group fingerprints in some cases. Since fingerprint size depends on the growth of the human body. This might be because persons of smaller age group with a body structure bigger than their normal growth have fingerprints bigger than their group and thus classified by the system to have come from the older class.

(4) Furthermore, the fingerprints of different age groups also vary in size and patterns as well as ridges and valley thickness. A male with a much higher body size may come with a higher ridge and valley thickness than normal and vice-versa resulting possibly in the gender mis-classifications.

\section{CONCLUSION}

A system that can predict or affirm the gender and age range of people is presented here through the use of Back Propagation Neural Network and DWT/PCA respectively. This was done judging from the Ridge Thickness to Valley Thickness Ratio (RTVTR) and Ridge Count (RC). Observation showed that females have it on the higher side in RTVTR and the males are favoured with a higher RC. Inaccuracies due to the observed mis-classifications in both gender and age could be because some people have much larger growth (body sizes) than their counterparts in sex group and age range.

\section{RECOMMENDATION}

Future research that will combine DNA analysis with the fingerprint ridge information might yield a better accuracy of both gender and age classification. And age classification system using the range of $1-3,4-6, . .19-21$ is desired to check the age range of subjects qualified for voting in State elections which is one of the motivations for this work. 


\section{ACKNOWLEDGEMENT}

We want to appreciation Prof. E.O. Omidiora of the Department of Computer Science and Engineering, Ladoke Akintola University of Technology, Ogbomoso, Nigeria, leader of my Biometric research group for his immense guidance.

\section{REFERENCES}

[1] Acree M.A. 1999. Is there a gender difference in fingerprint ridge density? Forensic Science International, 102: 35-44.

[2] Babler. 1991 Handbook of fingerprint recognition Springer Verlag.

[3] Badawi Ahmed, Mohamed Mahfouz, Rimon Tadross, Richard Jantz. 2008 Fingerprint- Based Gender Classification.

[4] Cappelli R., Lumini A., Maio D., and Maltoni D. 2007 "Fingerprint Image Reconstruction from Standard Templates", IEEE Transactions, Vol.29, pp.1489-1503.

[5] Cote Karine, Earls Christopher M, and. Lalumiere Martin L.2002 "Birth Order, Birth Interval, and Deviant Sexual Preferences Among Sex Offenders." Sexual Abuse: A Journal of Research and Treatment, Vol. 14, No. 1.

[6] Cummins, H. 1943. Fingerprints, Palms and soles.

[7] Falohun A.S., Oke A.O., Gbadamosi O.A 2015. Development of an Electronic Attendance Biometric Registration System. International Journal of Computer Trends and Technology (IJCTT) 27(3):126130, September 2015.

[8] Gu J., Zhou J., and Yang C. 2006 Fingerprint
Recognition by combining global structure and local clues.IEEETransactionsonImageProcessing,15(7):1924\{ 1964.

[9] Hall, J. A. Y. and Kimura D. 1994 "Dermatoglyphic Asymmetry and Sexual Orientation in Men." Behavioral Neuroscience, 108, 1203-1206,

[10] Hicklin R. Austin, Christopher L. Reedy, 2002 "Implications of the IDENT/IAFIS Image Quality Study for Visa Fingerprint Processing", Mitertek Systems (MTS).

[11] http://shodhganga.inflibnet.ac.in/bitstream/10603/6681/8 /09_chapter2 .pdf referenced on 14 Mar. 2015.

[12] Jain A.K, Sarat C. Dass, and Karthik Nandakumar. 2004 Soft Biometric Traits for Personal Recognition Systems Proceedings of International Conference on Biometric Authentication, LNCS 3072, pp. 731-738, Hong Kong.

[13] Komarinski Peter 2004 Automated Fingerprint Identification System (AFIS) Academy Press.

[14] Kralik M., Novotny V. (2003) Epidermal Ridge Breadth: An Indicator of Age and Sex in Paleodermatoglyphics. Variability and Evolution. Vol-11; 5-30.

[15] Maltoni D. Jain A.K., Maio D. and Prabhakar S. (2003) Handbook of Fingerprint Recognition. Springer, New York.

[16] Omidiora E.O.,Ojo O.,Yekini N.A.and Tubi T.O (2012) Analysis, Design and Implementation of Human Fingerprint Patterns System "Towards Age \& Gender Determination, Ridge Thickness To Valley Thickness Ratio (RTVTR) \& Ridge Count On Gender Detection (IJARAI) International Journal of Advanced Research in Artificial Intelligence, Vol. 1, No. 2, 2012 pp:57-63. 